\title{
A REMARK ON THE CONSTRUCTION OF NORMAL ELEMENTS IN CARTAN SUBALGEBRA
}

\section{HAITAO WAN}

School of Mathematics and Information Science

Weifang University

Weifang, 261061

P. R. China

e-mail: wht200805@163.com

\begin{abstract}
In this paper, we show that each Cartan subalgebra of finite-dimensional Lie algebra has normal elements by a simple way.
\end{abstract}

\section{Introduction and Main Result}

It is well known that a Cartan subalgebra can be constructed by a normal element. In turn, the converse of this statement is also true, i.e., each Cartan subalgebra has at least a normal element. In this paper, we give a simple and exquisite proof about this assertion.

Theorem 1.1. For each Cartan subalgebra of arbitrary finitedimensional Lie algebra, there must be a normal element. 


\section{Preliminaries}

Definition 2.1 ([1, p. 65, Definition 2.6.2]). Suppose $\hat{g}$ is a finitedimensional Lie algebra, $x \in \hat{g}$, and the multiplicity of $\lambda$ on the characteristic polynomial $\operatorname{det}\left(\lambda i d_{\hat{g}}-a d x\right)$ of $a d x$ is denoted by $n_{x}$. $n=\min \left\{n_{x} \mid x \in \hat{g}\right\}$ is call the rank of $\hat{g}$. If $x \in \hat{g}, n_{x}=n$, then $x$ is called normal element; $n_{x}>n$, then $x$ is called singular element.

Lemma 2.2. Suppose $\breve{b}$ is a Cartan subalgebra of finite-dimensional Lie algebra $\hat{g}, \Delta$ is the roots system of $\breve{b}$, then $x \in \breve{b}$ is the normal element of $\hat{g}$ if and only if $\forall \alpha \in \Delta, \alpha(x) \neq 0$.

Remark 2.3. Let $\hat{g}$ be a finite-dimensional Lie algebra on the number field $F$. If $x \in \hat{g}$ is a normal element, then $\eta \cdot x$ is also a normal element for any $\eta \in F$.

\section{Proof of Main Result}

Proof. Assume $\hat{g}$ is a finite-dimensional Lie algebra on the number field $F$ and $\breve{b}$ is a Cartan subalgebra of $\hat{g}$, and $\hat{g}$ can be divided as

$$
\hat{g}=\check{b}+\sum_{\alpha \in \Delta} \hat{g}_{\alpha}
$$

where $\Delta$ is the roots system and $\hat{g}_{\alpha}$ is the root subspace of $\hat{g}$. We can always select a group of bases in $\hat{g}$ marked as $v_{1}, v_{2}, \ldots, v_{n}$ such that $\forall x \in \breve{b}$ $\operatorname{adx}\left(v_{1}, v_{2}, \cdots, v_{n}\right)^{T}$

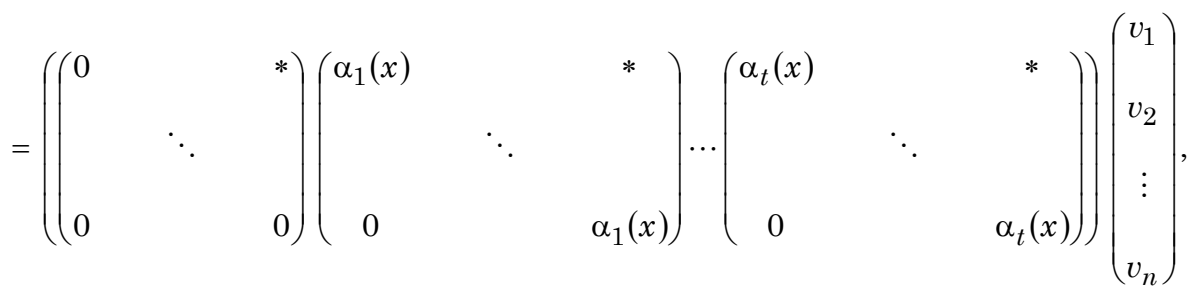


where $\alpha_{1}, \alpha_{2}, \ldots, \alpha_{t}$ are roots of $\breve{b}$. Then we obtain that the determinant of the matrix of linear transformation $\lambda I d_{\hat{g}}-a d x$ based on this group of bases is

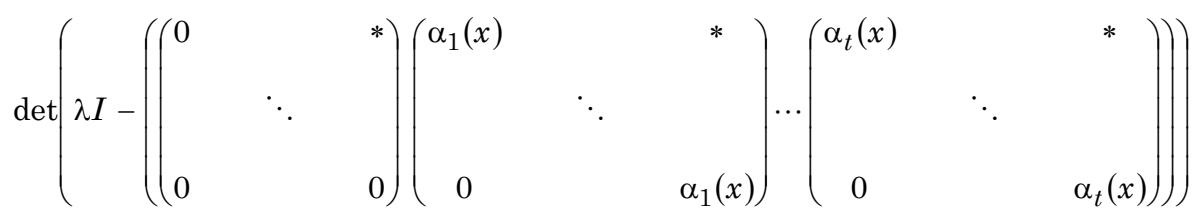

$=\lambda^{r_{0}} \times\left(\lambda-\alpha_{1}(x)\right)^{r_{1}}, \ldots,\left(\lambda-\alpha_{t}(x)\right)^{r_{t}}$,

where $r_{i}(i=0,1,2, \ldots, t)$ satisfy $\sum_{i=0}^{t} r_{i}=d$, and $d$ is the dimension of $\hat{g}$. We know $r_{0} \leqslant n_{x}$ from the definition of $n_{x}$. Next, we will prove that there exists $x_{0} \in \breve{b}$ such that

$$
\alpha_{\mathrm{i}}\left(x_{0}\right) \neq 0, \quad i=1,2, \ldots, t .
$$

If (3.1) is true, then it follows by Lemma 2.2 that Theorem 1.1 holds.

For $\alpha_{i} \neq 0, i=1,2, \ldots, t$, we can always find a element $x$ which belongs to $\breve{b}$ such that

$$
\alpha_{i_{1}}(x) \neq 0, \alpha_{i_{2}}(x) \neq 0, \ldots, \alpha_{i_{k}}(x) \neq 0, \quad k \leqslant t .
$$

If $k=t$, then the conclusion is obtained immediately. Otherwise, if $k<t$, we can get

$$
\alpha_{i_{j}}(x) \neq 0, j=1,2, \ldots, k, \alpha_{i_{k+l}}(x)=0, \quad l=1,2, \ldots, t-k .
$$

May as well suppose that

$$
\alpha_{i_{j}}(x)=\alpha_{j}(x), \alpha_{i_{k+l}}(x)=\alpha_{k+l}(x), j=1,2, \ldots, k, l=1,2, \ldots, t-k .
$$

Then we obtain

$$
\alpha_{1}(x) \neq 0, \alpha_{2}(x) \neq 0, \ldots, \alpha_{k}(x) \neq 0, \alpha_{k+1}(x)=0, \ldots, \alpha_{t}(x)=0 .
$$

Combining with $\alpha_{k+1} \neq 0$, we obtain that there exists $y \in \check{b}$ such that $\alpha_{k+1}(y) \neq 0$. 
If the following hold,

$$
\alpha_{i}(x+y) \neq 0, \quad i=1,2, \ldots, k,
$$

then we have

$$
\alpha_{i}(x+y) \neq 0, \quad i=1,2, \ldots, k+1 .
$$

If (3.3) is not true, then we can still find a element $x^{\prime} \in \breve{b}$ such that

$$
\alpha_{i}\left(x^{\prime}\right) \neq 0, \quad i=1,2, \ldots, k+1 .
$$

In fact, we may as well assume that

$$
\begin{aligned}
& \alpha_{1}(x+y) \neq 0, \alpha_{2}(x+y) \neq 0, \ldots, \alpha_{r}(x+y) \neq 0, \\
& \alpha_{r+1}(x+y)=0, \alpha_{r+2}(x+y)=0, \ldots, \alpha_{k}(x+y)=0, \quad 0 \leq r<k .
\end{aligned}
$$

Define

$$
A=\left\{\left|\alpha_{j}(y)\right| \neq 0 \mid j=1,2, \ldots, k\right\} .
$$

It follows by (3.2) and (3.4) that $|A| \geqslant k-r$.

Let

$$
\Lambda>\frac{\max \left\{\left|\alpha_{i}(x)\right|: i=1,2, \ldots, k\right\}}{\min A}, \text { and } x^{\prime}=x+\Lambda y,
$$

then we obtain $x^{\prime} \in \check{b}$ and

$$
\alpha_{\mathrm{i}}\left(x^{\prime}\right)=\alpha_{i}(x+\Lambda y)=\alpha_{i}(x)+\Lambda \alpha_{i}(y) \neq 0, \quad i=1,2, \ldots, k .
$$

Furthermore, we get $\alpha_{i}\left(x^{\prime}\right) \neq 0, i=1,2, \ldots, k+1$. Since there are finite members in $\Delta$, by repeating the above steps, we can always get $x_{0} \in \check{b}$ such that $\alpha_{i}\left(x_{0}\right) \neq 0, i=1,2, \ldots, t$.

\section{Reference}

[1] D. G. Meng, Introduction of Complex Semisimple Lie Algebras, First Ed., Peking University Press, Peking, 1998. 